\title{
Neuromagnetic correlates of visual motion coherence
}

\author{
J. E. Aspell, ${ }^{1, \star}$ T. Tanskanen ${ }^{2}$ and A. C. Hurlbert ${ }^{1, \dagger}$ \\ ${ }^{1}$ Department of Physiological Sciences, University of Newcastle, Newcastle Upon Tyne, UK \\ ${ }^{2}$ Brain Research Unit, Low Temperature Laboratory, Helsinki University of Technology, Espoo, Finland \\ Keywords: hMT+ complex, human, magnetoencephalography, random dot kinematogram, STS, timing
}

\begin{abstract}
In order to characterize cortical responses to coherent motion we use magnetoencephalography (MEG) to measure human brain activity that is modulated by the degree of global coherence in a visual motion stimulus. Five subjects passively viewed two-phase motion sequences of sparse random dot fields. In the first (incoherent) phase the dots moved in random directions; in the second (coherent) phase a variable percentage of dots moved uniformly in one direction while the others moved randomly. We show that: (i) visual-motion-evoked magnetic fields, measured with a whole-scalp neuromagnetometer, reveal two transient events, within which we identify two significant peaks - the 'ON-M220' peak approximately $220 \mathrm{~ms}$ after the onset of incoherent motion and the 'TR-M230' peak, approximately $230 \mathrm{~ms}$ after the transition from incoherent to coherent motion; (ii) in lateral occipital channels, the TR-M230 peak amplitude varies with the percentage of motion coherence; (iii) two main sources are active in response to the transition from incoherent to coherent motion, the human medial temporal area complex/V3 accessory area (hMT+/V3A) and the superior temporal sulcus (STS), and (iv) these distinct areas show a similar, significant dependence of response strength and latency on motion coherence.
\end{abstract}

\section{Introduction}

Visual motion provides important cues for image segmentation, via both motion discontinuities and motion grouping within regions (Braddick, 1993; Møller \& Hurlbert, 1997). A critical area for motion perception per se is the human MT complex (hMT+), given its likely homology with monkey medial temporal area (MT) (Zeki, 1980; Sereno, 1998), evidence from natural lesion studies (Vaina, 1994; Zihl et al., 1983) and functional imaging experiments (e.g. Tootell et al., 1995; Anderson et al., 1996; Patzwahl et al., 1996; Uusitalo et al., 1996; Vanni et al., 1997). But the extent to which MT contributes to the distinct processes involved in motion segmentation is not clear, nor is its relationship with other areas that signal surface formation based on other visual attributes.

There is evidence that MT neurons integrate discrete motion signals over space (Andersen, 1997; Qian \& Andersen, 1994; Movshon \& Newsome, 1996). For random dot kinematograms (RDKs), the firing rate of individual MT neurons increases with the percentage of coherent motion in the preferred direction in the classical receptive field, and predicts the monkey's performance on a direction discrimination task (Britten et al., 1992). Yet there are at least two distinct subtypes of MT neurons (Born \& Tootell, 1992): 'local-motion' neurons, whose responses decrease when the preferred motion stimulus extends into the inhibitory surround (Allman et al., 1985; Tanaka et al., 1986); and 'global-motion' neurons, whose responses increase as the preferred motion stimulus expands beyond the

Correspondence: Dr J. E. Aspell, *present address below.

E-mail: jane.aspell@psy.ox.ac.uk

*Present address: Department of Experimental Psychology, South Parks Road, Oxford, OX1 3UD, UK.

†Present address: School of Biology and Psychology, Institute of Neuroscience, University of Newcastle, Newcastle Upon Tyne, NE2 4HH, UK.

Received 22 July 2004, revised 26 July 2005, accepted 19 September 2005 receptive field (Born \& Tootell, 1992; Reppas et al., 1997). Localmotion neurons appear to outnumber global-motion neurons (Allman et al., 1985; Tanaka et al., 1986). For this reason, it remains unclear whether central stimuli larger than the 1-4 degree MT receptive field size near the fovea (Raiguel et al., 1995) would evoke a 'motionsurface' population response that increases with overall increasing coherence.

Several recent functional magnetic resonance imaging (fMRI) studies conclude that human MT - unlike V1 - is selectively activated by coherent motion in comparison with incoherent motion or dynamic noise (Heeger et al., 1999; Braddick et al., 2001), but there are conflicting reports (e.g. McKeefry et al., 1997; Lam et al., 2000). Furthermore, few studies have investigated whether the amplitude of neural responses in MT or other areas depends systematically on the strength of motion coherence (but see Patzwahl \& Zanker, 2000; Rees et al., 2000; Nakamura et al., 2003). Apparent discrepancies between these various studies suggest that MT activation may depend on particular stimulus parameters, such as the number and density of discrete motion elements and the overall size of the stimulus (see, e.g. Tadin et al., 2003), which influence the activation of distinct neuronal subtypes in MT. Studies of the effect of motion coherence on neuronal response latency have also failed to agree (compare Patzwahl \& Zanker, 2000 and Nakamura et al., 2003).

The aim of this study is to characterize cortical responses to coherent visual motion. We use magnetoencephalography (MEG), a technique that directly measures neuronal activity, in order to clarify the role of human MT and other brain areas in signalling coherent motion, and to relate our findings to known neuronal properties. We use high-contrast stimuli large and dense enough to activate 'globalmotion' MT neurons as well as 'local-motion' neurons. We also exploit the fact that MEG has a superior temporal resolution to fMRI and positron emission tomography (PET; milliseconds vs. seconds-tominutes) and is therefore the optimal technique to clarify the effect of 
motion coherence on the timing of neuronal responses. MEG also has superior spatial resolution to electroencephalography (reviewed in Hämäläinen et al., 1993), and therefore enables more accurate localization of sources underlying motion-evoked responses. A brief report of the present study appeared in Aspell et al. (2000).

\section{Materials and methods}

Five healthy right-handed volunteers (two males M1-M2, three females F1-F3; age range 22-41 years) participated in the MEG recordings. Structural MR brain images were obtained for all subjects, enabling anatomical source localization. The study was undertaken with the understanding and informed consent of each subject. The experimental procedures used in this study have been approved by the Helsinki Uusimaa Ethics Committees.

\section{Stimuli}

The stimulus was presented in a two-phase motion sequence, consisting of 400 limited lifetime white dots (luminance $23 \mathrm{~cd} / \mathrm{m}^{2}$ ), each $0.27 \mathrm{deg} \times 0.27 \mathrm{deg}$ square, randomly positioned in a $17.5 \mathrm{deg} \times 18 \mathrm{deg}$ black field (luminance $0.6 \mathrm{~cd} / \mathrm{m}^{2}$ ), with an average dot density of $1.27 \mathrm{dots} / \mathrm{deg}^{2}$. The stimulus was computergenerated by custom-made programs (on a Silicon Graphics Indy or PC) and projected onto a rear-projection screen at a viewing distance of $84 \mathrm{~cm}$, using a projector with Digital Light Processing TM technology (VistaProTM, Christie Digital Systems Inc., Cypress, CA, USA). The projector hosts three digital micromirror panels; thus the luminance onsets and offsets are symmetric and abrupt, and all three colours are drawn simultaneously. Subjects passively viewed the motion sequences while maintaining fixation on a central white cross.

Each motion sequence consisted of two phases: an 'incoherent' phase of $883 \mathrm{~ms}$, followed immediately by a 'coherent' phase of $400 \mathrm{~ms}$. We chose this two-phase motion sequence because it allowed us to distinguish between a general motion onset response and a coherent motion onset response. The long duration of the incoherent phase insures that the general motion onset response, which occurs in response to the onset of incoherent motion, is well over before the transient response to the change to coherent motion begins; the responses are therefore separable. The long duration is also necessary to prevent motion adaptation. Bach \& Ullrich (1994) showed that a short duty cycle (i.e. a stimulus with a relatively longer stationary/incoherent phase than moving/coherent phase) emphasizes the motion evoked N200 response. Our pilot studies also found that relatively long incoherent phases produced larger amplitude responses to coherent motion onset.

During the incoherent phase, all dots moved in random directions at a speed of $8 \mathrm{deg} / \mathrm{s}$. During the coherent phase, a variable percentage of randomly chosen dots moved uniformly in one direction (left or right horizontally) against the continued random motion of the others. To prevent the subject tracking individual dots, the program assigned $5 \%$ of dots on each frame a new direction selected at random from either the 'incoherent' distribution (uniform in direction from 0 to 360 degrees) or 'coherent' distribution (single in direction, either left or right), with the probability of selection from the coherent distribution set by the proportion of coherent dots in the current phase. At the displayed frame rate of $60 \mathrm{~Hz}$, all dots in a given display would be replaced on average between two and three times in the incoherent sequence, and at least once during the coherent phase. Dots remained static on the screen in their stopping position between trials, with an interstimulus interval of $2.5 \mathrm{~s}$ (see Fig. 1).

The proportion of coherent dots in the second phase and the direction of coherent motion (left or right) were varied across conditions. Each run lasted approximately $10 \mathrm{~min}$ and comprised approximately 35 trials each of four conditions: 100\%-right, 100\%left, and the left and right directions of a test coherence level, either 40,60 or $80 \%$. (Conditions are hereafter labelled by the coherence level of the coherent phase.) The presentation order of left and right directions was randomised. Each subject performed 4-6 runs per session, with two runs per test coherence block, tested consecutively in ascending order (i.e. 100-40, 100-60, 100-80). Subjects performed a maximum of two sessions to complete the experiment. The $60 \%$ level was not tested on two subjects.

\section{MEG recordings}

Subjects passively viewed the stimuli while seated in a magnetically shielded room, their heads positioned in the helmet of a 306-channel Vectorview ${ }^{\mathrm{TM}}$ neuromagnetometer (Neuromag Ltd, Finland). Before the MEG recordings, four head position marker coils were attached to the subject's scalp. The positions of the coils and of three anatomical landmarks (nasion and points immediately anterior to the ear canals) were measured with a 3D digitizer (3Space FastrakTM, Polhemus Inc., Colchester, VT, USA). At the beginning of each recording block, the position of the subject's head with respect to the sensor array was determined by feeding current to the coils. This information was afterwards used to combine the measured neuromagnetic signals with the subjects' anatomical MRIs.

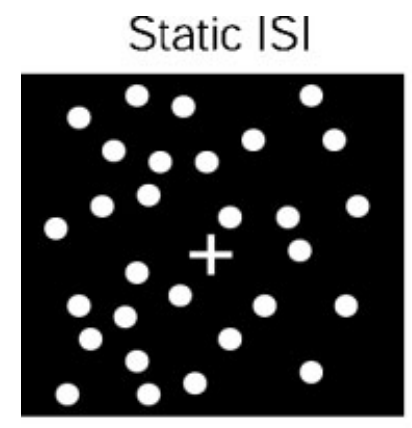

$2500 \mathrm{msec}$

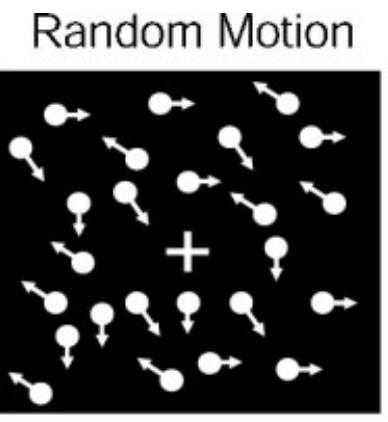

$883 \mathrm{msec}$
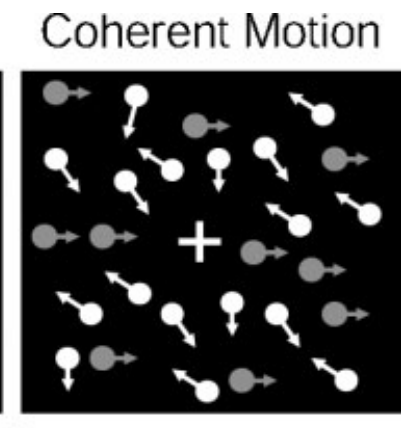

$400 \mathrm{msec}$

FIG. 1. Schematic illustration of visual motion stimulus showing the durations of the interstimulus interval (ISI) and the two different motion phases. See main text for details. 
The cortical signals were measured at 102 head surface positions, with two orthogonal planar gradiometers and one magnetometer at each. The signals were initially bandpass filtered between 0.1 and $172 \mathrm{~Hz}$ and digitized at $0.6 \mathrm{kHz}$. Horizontal and vertical electrooculograms were recorded simultaneously with MEG and trials contaminated by eye movements or blinks were rejected.

\section{Data analysis}

The averaged signals were preprocessed by omitting noisy channels, attenuating environmental noise by projecting out noise subspaces determined from empty room measurements (Parkkonen et al., 1999), and applying a digital low pass filter at $40 \mathrm{~Hz}$. The responses to rightward and leftward motion did not differ from each other and were therefore averaged together, resulting in approximately 100 trials per average. (Note that the $100 \%$ conditions from distinct test coherence level runs were not averaged together.)

We restricted our analysis to the 204 gradiometer channels, which give the largest signals just above a locally activated brain area, and therefore allow us to use the locations with largest signals as first estimates of the activated brain areas. To discount sustained activity elicited by the first phase of incoherent motion and to focus on sources activated by the transition to coherent motion, we set the baseline for signal analysis to the pre-transition period ( $700-883 \mathrm{~ms}$ ). (This choice of baseline setting can be justified because for all stimuli, the incoherent motion phase was identical.) We used a spherical volume conductor model, fitted to the posterior portion of the brain, encompassing occipital, parietal and temporal regions. The sources of the evoked responses were identified by searching for equivalent current dipoles (ECDs) during restricted time periods after the transition from incoherent to coherent motion. We focused on signals generated in the posterior parts of the brain. Single dipoles were searched by a least-squares fit to explain the responses on 15-32 channels centred over the channels with the largest motion-transition responses. Only ECDs that explained at least $80 \%$ of the local field variance on the selected sensors were accepted. The contribution of the fitted source was then projected out of the measured signals using the Signal Space Projection method (Uusitalo \& Ilmoniemi, 1997) and where possible, new dipole sources were fit to the part of the data not explained by the first dipole. The whole sequence was repeated and dipoles added until no signals above noise level remained unexplained by the source model. All dipoles found in this way were then used to explain the nonprojected data to reveal source strengths as a function of time.

To examine explicitly the dependence of source strength on test coherence level, we fitted dipoles to the $100 \%$ condition in each distinct test coherence block, and then used these dipoles to explain the responses in the respective paired test coherence condition. Thus, for each subject, we obtained at least two distinct ' $100 \%$ dipoles', for the 100-40 and 100-80 blocks. The locations of the $100 \%$ dipoles did not vary significantly, and in each case, the ' $100 \%$ dipole' explained as much or more variance than the ECD for the paired condition.

\section{Results}

The averaged evoked responses over the full motion sequence showed several features across subjects (see Fig. 2). In occipital and lateral channels, two transient events (defined by activity with absolute magnitude greater than three standard deviations of the prestimulus signal variation) were obvious, around the onset and motion-transition times of the stimulus. The first event was a triphasic complex that began approximately $80 \mathrm{~ms}$ after the onset of incoherent motion. Its middle deflection (of opposite polarity to the first deflection) was typically the largest of the three, and peaked at approximately $220 \mathrm{~ms}$ (171-238 ms, across subjects and coherence levels). This we assume to be the magnetic counterpart of the classic electroencephalgraphic N200 response (see Discussion), and we term it the ON-M220 peak. The second event was a predominantly monophasic deflection, with the same polarity as the ON-M220 peak. This peak, which we term the TR-M230, occurred at approximately $230 \mathrm{~ms}$ after the transition from incoherent to coherent motion (172-256 ms across subjects and coherence levels). Both ON-M220 and TR-M230 were dominant in the temporo-occipital and occipital regions. In all but one subject (F3), there was a marked hemispheric asymmetry in the TR-M230 amplitude, it being much larger in several lateral occipital channels in one hemisphere than the other.

Most importantly, for all subjects, the amplitude of TR-M230 clearly varied with the test coherence level in several lateral occipital channels. Figure 2 illustrates these findings for one subject. The TRM230 peak varies with test coherence level in lateral occipital channels, whereas there is no consistent variation either in the ON-220 peak or in the TR-M230 peak in response to the $100 \%$ condition from different test coherence blocks. In further analysis, we therefore focused only on those regions in which the TR-M230 peak varied with test coherence level.

Insets in Fig. 3 illustrate for subject F2 the magnetic field patterns at the peaks of the TR-M230 responses, and corresponding source areas superimposed on her MR images. The sources are located in the $\mathrm{hMT}+/ \mathrm{V} 3 \mathrm{~A}$ and superior temporal sulcus (STS) regions. For both sources, there was a clear peak in activity around $230 \mathrm{~ms}$ after the transition from incoherent to coherent motion. The amplitude of this peak was highest for the $100 \%$ condition, and decreased for the $80 \%$ and $40 \%$ conditions.

In all subjects, source modelling around the TR-M230 peak revealed activation of several distinct cortical regions. Reliable sources were found in three regions, with the following Talairach co-ordinates (mean $\pm \mathrm{SEM}$ ): hMT $+/ \mathrm{V} 3 \mathrm{~A}$ (5/5 subjects; left hemisphere $-33 \pm 3,-68 \pm 4,12 \pm 3$; right hemisphere $38 \pm 4,-71 \pm 3$, $6 \pm 1)$, STS (4/5 subjects; right hemisphere $44 \pm 4,-40 \pm 6,13 \pm 4$; left hemisphere $-56,-25,8)$, and intraparietal sulcus (IPS) $(1 / 5$ subjects; right hemisphere $25,-63,45)$. Figure 4 shows the locations of the hMT $+/ \mathrm{V} 3 \mathrm{~A}$ and STS sources for all five subjects overlaid on a single MR brain image (from subject F1), transformed to standard space using FLIRT, FMRIB's Linear Image Registration Tool (Jenkinson \& Smith, 2001; Jenkinson et al., 2002). See Table 1 for the Talairach co-ordinates of these sources in individual subjects. All sources were strongly lateralized in each subject, except for $\mathrm{hMT}+/ \mathrm{V} 3 \mathrm{~A}$ in subject $\mathrm{F} 3$ who showed bilateral hMT+/V3A activity. In two female subjects (F1 and F2), hMT $+/ \mathrm{V} 3 \mathrm{~A}$ activity was detected only in the left hemisphere, and in the two male subjects, hMT $+/ \mathrm{V} 3 \mathrm{~A}$ activity was detected only in the right hemisphere. STS was also lateralized, being dominant on the right in three out of four subjects.

The amplitude of the hMT+/V3A source strongly depended on the motion coherence of the second phase, in general increasing with increasing motion coherence (Fig. 5). An unbalanced, multiway, repeated measures ANOVA (subject $\times$ coherence) revealed significant main effects for coherence level $\left(F_{2,6}=11.54 ; P=0.0088\right)$ and subject $\left(F_{5,6}=40.17 ; \quad P=0.0002\right)$, but no interaction effect $\left(F_{10,6}=1.29 ; P=0.393\right)$. Differences between means were compared using the Bonferroni post hoc test; significant differences in peak amplitude were found between $100 \%$ and $40 \%(P=0.009)$. A 

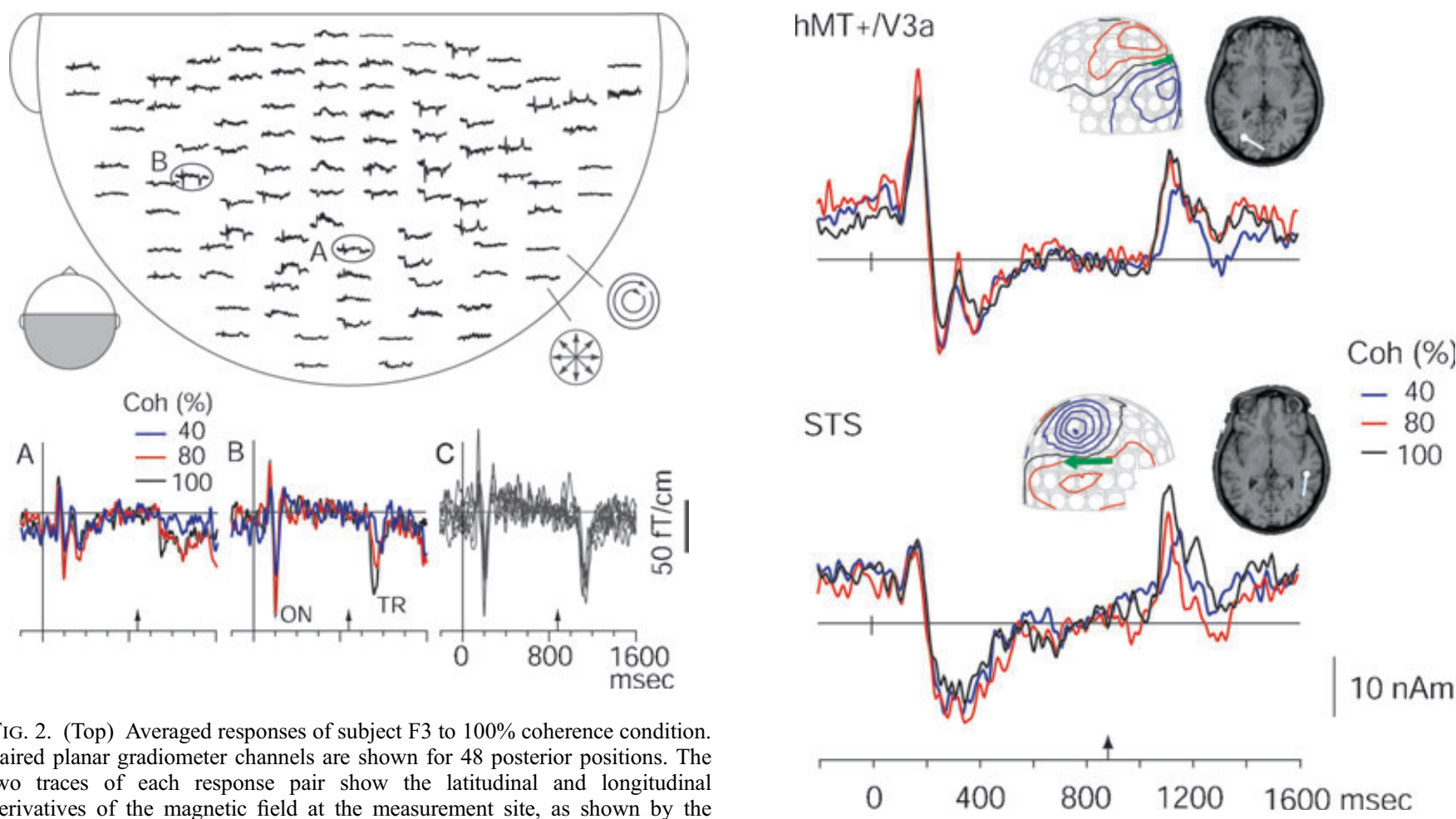

FIG. 2. (Top) Averaged responses of subject F3 to $100 \%$ coherence condition. Paired planar gradiometer channels are shown for 48 posterior positions. The two traces of each response pair show the latitudinal and longitudinal derivatives of the magnetic field at the measurement site, as shown by the symbols at the bottom right. (Bottom) Responses to different coherence conditions in demonstrative channels over the mid-occipital (A) and lateral occipital (B) region. ON, onset response; TR, transition (to coherent motion) response. All responses are baselined to the time period $700-883 \mathrm{~ms}$ (i.e. to just before motion transition). Arrow marks the time point of transition to coherent motion. (C) Superimposed responses in channel B to six different presentations of $100 \%$ coherence condition; three to leftwards motion only; three to rightwards motion only. Each response is averaged over 43 to 58 trials.

trend analysis revealed a significant linear trend for coherence $(P=0.003)$.

The STS source activity showed a similar dependence on motion coherence (Fig. 5) and an unbalanced, multiway, repeated measures ANOVA revealed significant main effects for coherence level $\left(F_{2,4}=9.9 ; \quad P=0.028\right)$ and near-significant effects for subject $\left(F_{3,4}=6.03 ; P=0.058\right)$, but no interaction effect $\left(F_{6,4}=2.82\right.$; $P=0.1668$ ). Significant differences between means (using the Bonferroni post hoc test) were found between $100 \%$ and $40 \%$ $(P=0.035)$. A trend analysis revealed a significant linear trend for coherence $(P=0.012)$.

The peak latency for each dipole was measured as the time at which its maximum activity (following the onset of coherent motion at $883 \mathrm{~ms}$ ) occurred in the multidipole fit. For all five subjects, the peak latency of the TR-M230 decreased with increasing motion coherence (Fig. 6), for areas hMT $+/ \mathrm{V} 3 \mathrm{~A}$ and STS or IPS. For hMT $+/ \mathrm{V} 3 \mathrm{~A}$ response latency, a two-way unbalanced ANOVA revealed a significant main effect for coherence level $\left(F_{2,6}=17.17 ; P=0.0033\right)$ but not for subject $\left(F_{5,6}=4.1 ; P=0.06\right)$, and there was no interaction effect $\left(F_{10,6}=1.07 ; P=0.49\right)$. Significant differences between means (using the Bonferroni post hoc test) were found between $100 \%$ and $40 \%(P=0.003)$ and between $80 \%$ and $40 \%(P=0.006)$. A trend analysis revealed a significant linear trend for coherence $(P=0.001)$. Similarly, for STS latency, ANOVA revealed significant main effects for coherence level $\left(F_{2,4}=7.54 ; P=0.044\right)$, and subject $\left(F_{3,4}=7.28\right.$; $P=0.043)$, but no interaction $\operatorname{effect}\left(F_{6,4}=0.51 ; P=0.782\right)$. A trend analysis revealed a significant linear trend for coherence $(P=0.045)$. No significant difference between $\mathrm{hMT}+/ \mathrm{V} 3 \mathrm{~A}$ and STS latencies across coherence levels was found $\left(F_{1,34}=1.01 ; P=0.314\right)$.

FIG. 3. Amplitudes of the MT+ and STS sources of subject F2 as a function of time for different coherence conditions. Arrow marks the timepoint of transition to coherent motion. Insets show magnetic field patterns at $t=1120 \mathrm{~ms}$ during presentation of $100 \%$ condition and corresponding source locations superimposed on structural MRIs. Averaged responses were baselined to $183 \mathrm{~ms}$ preceding transition to coherent motion.
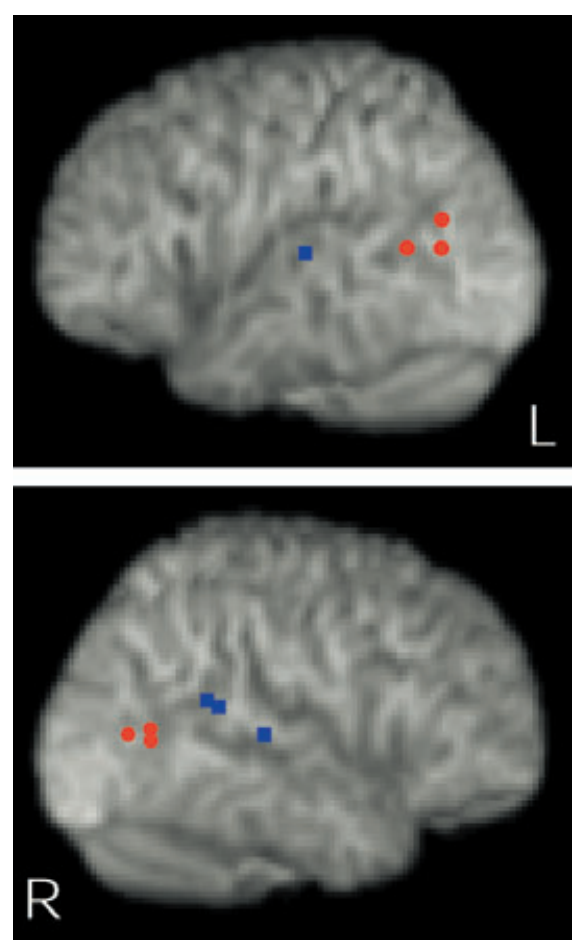

FIG. 4. Location of STS (blue squares) and hMT+/V3A sources (red circles) in R, right hemisphere and L, left hemisphere. The six hMT/V3A sources displayed are from all subjects and include two sources from subject F3 who showed bilateral activation. Rendered image of standard space brain (from subject F1) was created with MRIcro software (Rorden \& Brett, 2000). 


\section{Discussion}

MEG reveals several sources $-\mathrm{hMT}+/ \mathrm{V} 3 \mathrm{~A}$, STS and IPS (in only one subject) - that respond to the transition from incoherent to coherent motion, consistent with recent fMRI and MEG studies (Heeger et al., 1999; Rees et al., 2000; Braddick et al., 2001; Nakamura et al., 2003). The activity of these sources varies with coherence.

\section{$h M T+/ V 3 A$}

The Talairach co-ordinates of the motion sensitive sources identified in the present study are consistent with those previously reported for motion areas (e.g. Zeki et al., 1991; Watson et al., 1993; Tootell et al., 1995; Rees et al., 2000). The mean co-ordinates for human MT derived from these studies are 44, $-68,3$ (right hemisphere) and -42 , $-71,2$ (left hemisphere). The mean distances of hMT $+/ \mathrm{V} 3 \mathrm{~A}$ dipoles (Table 1) from these co-ordinates are, for right $\mathrm{hMT}+/ \mathrm{V} 3 \mathrm{~A}, 5,3$, $3 \mathrm{~mm}$, and for left $\mathrm{hMT}+/ \mathrm{V} 3 \mathrm{~A}, 9,5,9 \mathrm{~mm}$. The nearby motion sensitive area V3A, with co-ordinates (from Tootell et al., 1997) ranging from $\pm 29,-86,-16$ (central representation of $\mathrm{V} 3 \mathrm{~A}$ ) to \pm 14 , $-84,19$ (peripheral representation of $\mathrm{V} 3 \mathrm{~A}$ ), is elongated along the

TABLE 1. Talairach co-ordinates of ECDs for TR-M230, averaged across the several ' $100 \%$ dipoles' for each subject (see text)

\begin{tabular}{|c|c|c|c|c|c|c|}
\hline \multirow[b]{2}{*}{ Subject } & \multicolumn{3}{|c|}{$\mathrm{hMT}+/ \mathrm{V} 3 \mathrm{~A}$} & \multicolumn{3}{|l|}{ STS } \\
\hline & $x$ & $y$ & $z$ & $x$ & $y$ & $z$ \\
\hline $\mathrm{F} 1$ & -38.3 & -59.5 & 8.8 & 38.2 & -43.6 & 15.6 \\
\hline $\mathrm{F} 2$ & -26.9 & -71.2 & 17.6 & 51.0 & -29.4 & 6.1 \\
\hline F3 & -34.2 & -72.6 & 8.5 & & & \\
\hline F3 & 41.9 & -77.1 & 5.5 & & & \\
\hline M1 & 42.9 & -67.5 & 4.6 & 43.2 & -48.1 & 17.7 \\
\hline M2 & 30.4 & -68.6 & 7.4 & -56.0 & -24.7 & 7.5 \\
\hline
\end{tabular}

Subject F3 did not show STS activation but had an active source in the inferior parietal sulcus (co-ordinates 25.4, -63.4, 44.6).

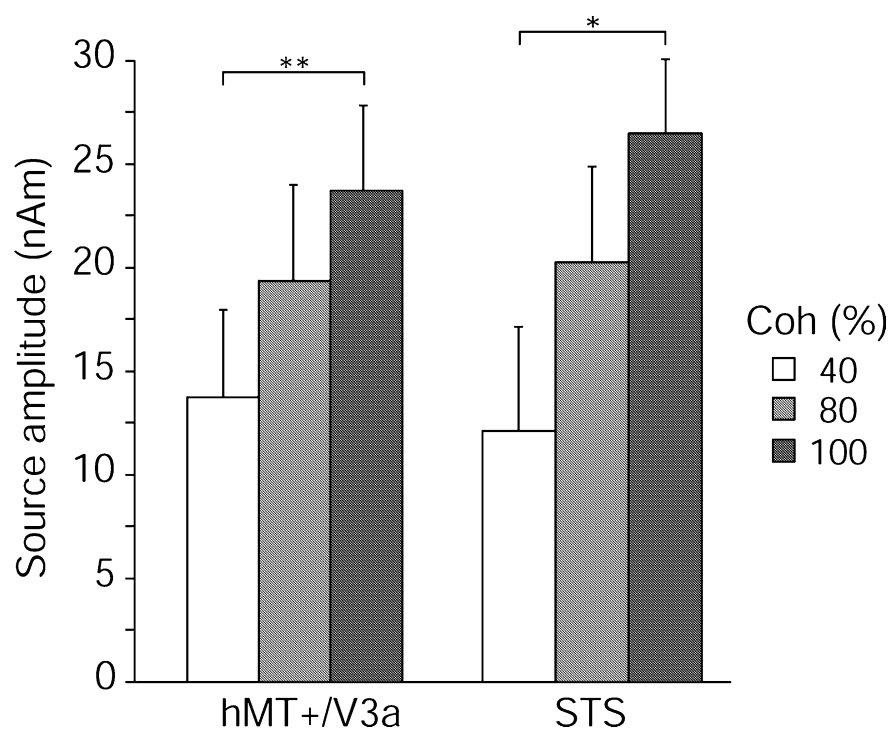

FIG. 5. Mean (+ SEM) source amplitudes of hMT $+/ \mathrm{V} 3 \mathrm{~A}$ and STS cortices during the motion transition peak for different coherence levels. $\left({ }^{*} P<0.05\right.$, $\left.{ }^{*} * P<0.01\right)$. The correlations between the normalized source amplitudes and motion coherence were $0.77(P<0.005)$ and $0.86(P<0.005)$ for the $\mathrm{hMT}+/ \mathrm{V} 3 \mathrm{~A}$ and STS sources, respectively.
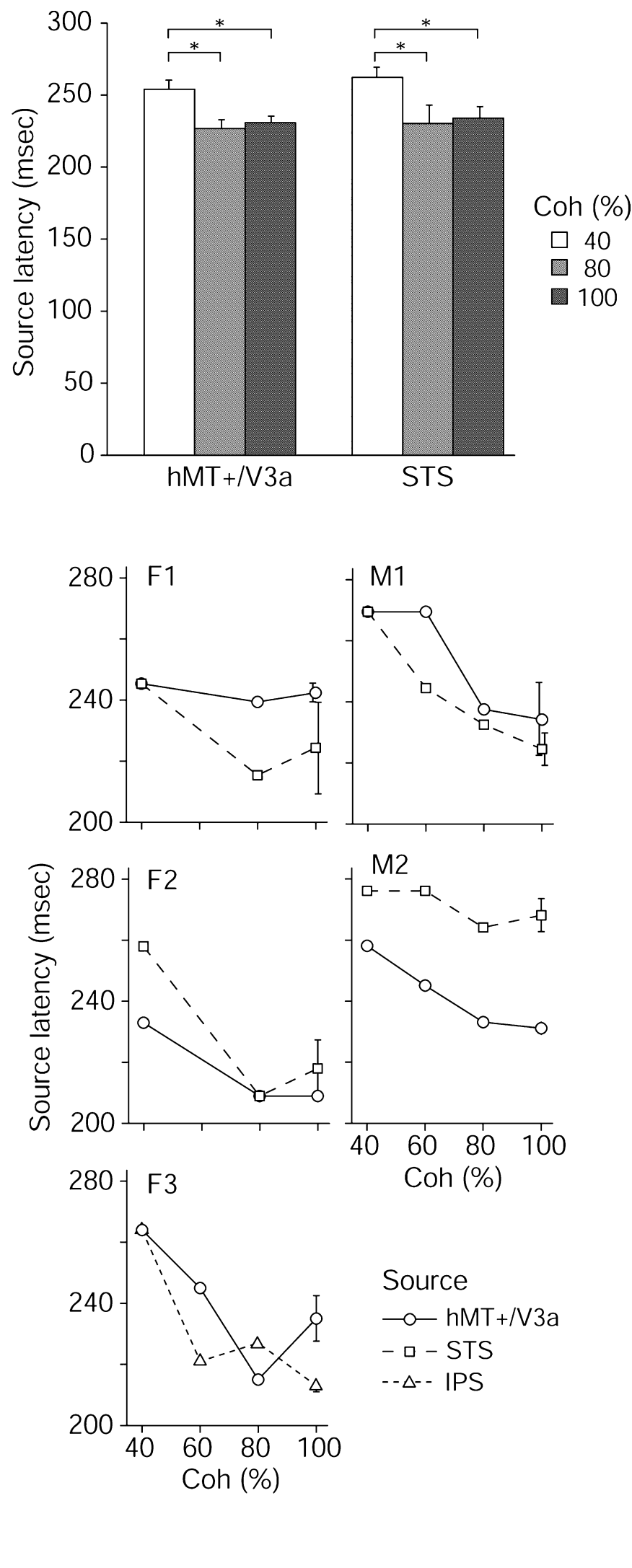

FIG. 6. (Top) Mean (+ SEM) peak latencies of the hMT $+/$ V3A and STS sources after motion transition as a function of motion coherence. $\left({ }^{*} P<0.05\right)$. (Bottom) Subplots show results for individual subjects; distinct lines within subplots represent distinct sources. Error bars show SEM for the $100 \%$ conditions. 
superior-inferior axis of the brain. The source analysis in this study does not permit reliable differentiation between the hMT complex and $\mathrm{V} 3 \mathrm{~A}$, and for this reason we refer to the source as hMT $+/ \mathrm{V} 3 \mathrm{~A}$.

The activity of hMT $+/ \mathrm{V} 3 \mathrm{~A}$ in general increased monotonically with increasing coherence in all subjects. This suggests an accompanying increase in the number of MT neurons that are firing in synchrony. What does this result tell us about neuronal properties in MT?

Single unit animal studies indicate that increasing coherence causes the response of 'local-motion' or 'opponent-surround' MT neurons to decrease (Allman et al., 1985; Tanaka et al., 1986), but the response of 'global-motion' neurons to increase (Reppas et al., 1997). Thus, if the activity of 'opponent-surround' neurons were to dominate the population response in human MT, we might expect the population response to decrease with increasing motion coherence. Indeed, single unit studies suggest that opponent-surround neurons do outnumber 'global-motion' neurons (Allman et al., 1985; Tanaka et al., 1986; Xiao et al., 1997); for example, Bradley \& Andersen (1998) report that approximately $81 \%$ of MT neurons evince direction-specific surround effects, and of these, approximately $88 \%$ show suppression rather than excitation in response to the preferred direction.

The population response should depend not only on the relative number of distinct neuronal types, but also on the effectiveness of the stimuli for each type. Stimulus size is a crucial factor. Classical receptive fields of local-motion neurons increase from between one and two degrees at the fovea to above 15 degrees at 26 degrees eccentricity (Raiguel et al., 1995), while the direction-specific surround effects may occur up to eight degrees beyond the centre, with maximal effects typically occurring at 1.5 times the centre radius (Xiao et al., 1997). Thus, near-central stimuli larger than a few degrees may be expected to recruit significant suppressive surrounds. For an RDK stimulus, as the number of coherent moving dots increases, increasing activation of the centre response may be counterbalanced by increasing suppression from the surround, and there may be no change in the population response. On the other hand, for small stimuli that fit the classical receptive field sizes at the stimulated eccentricity, the population response of 'local-motion' neurons would be expected to increase with increasing coherence. Accordingly, Rees et al. (2000) found a roughly linear increase in the fMRI blood oxygenation level dependent (BOLD) response of MT with increasing coherence in relatively small stimuli (4 degrees in diameter) displayed 4 degrees from the fovea, and successfully predicted the results with a model of neuronal firing using centre-only receptive fields without surround antagonism. Conversely, McKeefry et al. (1997) found using PET that hMT + was activated more strongly by incoherent than coherent motion relative to static patterns, for relatively large (20 degrees $\times 26$ degrees) stimuli. For these stimuli, McKeefry et al. (1997) argue that the suppressive surround response of local-motion neurons may dominate the population response. Shulman et al. (1999) also argue that they found enhanced activations (using PET) to motion-defined gratings in hMT+ and other early visual areas for the same reason. Stimulus size was therefore shown to be a very important factor in determining the population response of MT neurons to coherent motion stimuli.

In addition to stimulus size, the number, area coverage and contrast of the discrete motion elements (the dots in an RDK) may also regulate the effectiveness of the motion stimuli. As Braddick et al. (2001) argued, significant activation of hMT + by coherent motion may arise only when the number of discrete dots visible to individual neuronal receptive fields is large enough to allow for summation. Tadin et al. (2003) found that increasing the size of low-contrast or high-noise motion stimuli increases their direction discriminability, whereas increasing the size of high-contrast or low-noise stimuli decreases their discriminability. Tadin et al. (2003) argued that 'localmotion' neurons are responsible for these perceptual phenomena, and that 'weak' motion stimuli preferentially evoke spatial summation over spatial suppression. It may be therefore that there is an optimal range of dot-density and area coverage for RDKs within which the stimuli are sufficiently weak to evoke spatial summation yet not sufficiently powerful to evoke suppression, for 'local-motion' neurons.

On the other hand, if 'global motion' or 'facilitatory-surround' neurons were to dominate the population response, we would expect it to increase with increasing motion coherence even for relatively large or strong motion stimuli. Our finding fits this expectation, and is in line with other human studies using fMRI (Braddick et al., 2000), EEG (Niedeggen \& Wist, 1998, 1999; Patzwahl \& Zanker, 2000) and MEG (Nakamura et al., 2003). The results of our study are also qualitatively similar to those of Rees et al. (2000), but the considerable differences in dot density and stimulus size suggest that different neuronal mechanisms may underlie the similarity. Whereas Rees et al. (2000) used small, very low density (approximately $2 \%$ area coverage, with 20 discrete dots $/ \mathrm{deg}^{2}$ ) stimuli, we used large (18 degrees in diameter), moderate-density (approximately $10 \%$ area coverage, with approximately 1.3 discrete dots $/ \mathrm{deg}^{2}$ ) central stimuli. On the above arguments, one would expect the two different stimuli to elicit different responses from 'local-motion' neurons with large, nonclassical suppressive surrounds, with ours being nonoptimal for the latter (Raiguel et al., 1995). On the other hand, our stimuli are similar in size and area coverage, but much larger in dot number than those of McKeefry et al. (1997) (approximately 8.5\% dot density, with 0.19 discrete dots $/ \mathrm{deg}^{2}$ ), and therefore would be expected to provide an even stronger stimulus to the suppressive surrounds of 'localmotion' neurons. Yet, using even greater dot numbers and area coverage, Braddick et al. (2001) also found greater activation with fMRI to coherent motion compared with dynamic noise in $\mathrm{hMT}+$, V3A, STS, and IPS. Thus, we conclude that the increase in population activity that we recorded with increasing coherence reflects a dominant response from 'global-motion' neurons, which are sufficiently stimulated by our relatively large and dense stimuli to overcome the suppressive surround activity of 'local-motion' neurons.

This conclusion is supported by other recent MEG studies. Lam et al. (2000) found for a moderate-sized (10 deg $\times 10 \mathrm{deg})$, moderately dot-dense stimulus that incoherent and coherent motion activated presumptive MT equally strongly, whereas Nakamura et al. (2003) found for a very large $(50 \mathrm{deg} \times 60 \mathrm{deg})$, relatively low-density stimulus that the amplitude of MT activity increased with increasing coherence.

The extrapolation from single unit measurements in animals to population measurements with functional imaging in humans is evidently not trivial. For example, where neural codes are sparse and individual tuning curves broad and multidimensional, population activity as measured by fMRI will be little influenced by the firing of the few neurons that are tuned most strongly to the stimulus (see, e.g. Scannell \& Young, 1999). Nonetheless, direct comparison of neural activity and blood oxygenation level dependent (BOLD) responses in monkey visual cortex demonstrate that local neuronal field potentials correlate with the haemodynamic response (Logothetis et al., 2001).

While MEG provides a more direct measure of neural activity than does fMRI, it too is able only to detect the activity of thousands of synchronous neurons, and in this study we considered only the timelocked averages of such signals. Comparison of single cell firing rates with MEG signals is therefore not a straightforward task, even so, our results strongly support the conclusion that the activity of 'globalmotion' neurons in $\mathrm{hMT}+$ dominates the population response to relatively large, coherent-motion stimuli. 


\section{STS}

STS activation was detected in four out of five subjects and the response in this area increased with increasing motion coherence (Fig. 5). STS is a large and functionally diverse area; previous PET and $\mathrm{PMRI}$ studies have found that STS is activated by visual motion (Ahlfors et al., 1999) - including coherent visual motion (Dupont et al., 1994; Shulman et al., 1998; Braddick et al., 2000), 'biological motion' (Oram \& Perrett, 1994; Vaina et al., 2001), and motion of other individuals (Allison et al., 2000) - and includes an area that is preferentially activated by moving mouths and lips (Puce et al., 1998). The STS region in monkeys receives inputs from multiple sensory modalities (Bruce et al., 1981) and may be involved in cross-modal integration.

STS also appears to be involved in the extraction of form from motion; human STS has been shown to integrate form and motion (Puce et al., 2003; Schoenfeld et al., 2003) and this area is well situated to provide a link between motion processing areas and object identification areas in dorsal and ventral streams, respectively (Felleman \& Van Essen, 1991; Merigan \& Maunsell, 1993). Dupont et al. (1994) suggest that a region of STS (Talairach co-ordinates 52, $-38,16)$ similar to the areas reported here (mean co-ordinates of the right STS source $44,-40,13$ ) is the human homologue of monkey parieto-vestibular cortex (PIVC). In monkeys PIVC responds to uniform visual motion and receives input from vestibular cortex (Grüsser et al., 1990).

\section{Lateralization}

One intriguing feature of these results is the marked lateralization of the evoked response in $\mathrm{hMT}+/ \mathrm{V} 3 \mathrm{~A}$ and STS in most subjects. Bilateral activation of the presumed human MT+ complex has been reported (Dupont et al., 1994; Rees et al., 2000), but there is evidence for the lateralization of the motion response (Probst et al., 1993; Uusitalo et al., 1996; Niedeggen \& Wist, 1998, 1999). An extensive study into the hemispheric asymmetry of motion VEPs (Kubova et al., 1990) found that in $60 \%$ of 80 subjects the maximum amplitude of $\mathrm{N} 2$ was recorded at the electrode over the right hemisphere. Left hemisphere dominance was seen in only $20 \%$ of the subjects. In the present study, for three subjects the dominant hMT+/V3A and STS sources are in different hemispheres to each other, and for one subject, in the same hemisphere.

\section{Timing}

We found that the peak latency of the TR-M230 source decreased with increasing motion coherence for $\mathrm{hMT}+/ \mathrm{V} 3 \mathrm{~A}$, STS and IPS. While this finding is inconsistent with the nonsignificant increase in mean peak latencies of the MEG response to increasing motion coherence reported by Nakamura et al. (2003), it is in line with results from an earlier study. Using EEG, Patzwahl \& Zanker (2000) found an approximately linear relationship between reaction time and the latency of a transient component at $\sim 230 \mathrm{~ms}$ in a direction discrimination experiment. The increased latency at lower coherence levels may reflect the increased time necessary for temporal integration of motion signals to achieve the required level of confidence for an accurate response to be generated (Snowden \& Braddick, 1991; Britten et al., 1992).

As Lam et al. (2000) argued, the latency of the magnetic evoked response may reflect not only the latency of the individual neuronal evoked responses but also the time needed to synchronize multiple neuronal responses (see also Okada et al., 1997). If it is assumed that each neuronal element under consideration produces a PSP-type waveform, increasing synchrony results in a shortening of the peak latency, as well as an increase in the sum response amplitude (Hari, 1990). Hence the increased latency in response to the transition to $40 \%$ motion coherence might reflect the greater amount of time required to synchronize the weak activity of neurons with similar preferred directions at scattered locations across the visual field. For 100\% coherent motion, individual neurons whose preferred direction matches the coherent motion direction will be more strongly activated as they will sum the preferred direction across their whole receptive field.

For all subjects except one (M2) the latencies of TR-M230 overlapped for the different sources. Given that IPS and STS are considered to be higher in the hierarchy of cortical areas than $\mathrm{hMT}+/ \mathrm{V} 3 \mathrm{~A}$, it is perhaps surprising that the latencies of TR-M230 do not differ between these areas. Braddick et al. (2001) also found activation of STS and IPS in response to coherent motion, using fMRI, but attributed this to a feed-forward effect. The clear implication is that the signal should arrive later in the anterior systems.

Our $\sim 200 \mathrm{~ms}$ latency for a motion onset response agrees well with a large body of EEG and MEG studies on visual motion that define the 'N200 peak' to motion onset (Kuba \& Kubova, 1992; Bach \& Ullrich, 1994; Spileers et al., 1996; Skrandies et al., 1998; Hoffman et al., 1999; Patzwahl \& Zanker, 2000; Nakamura et al., 2003). The response to coherent motion onset that we measure is rather late to correspond to the processing in the 'feedforward sweep' of activation (Lamme \& Roelfsema, 2000) namely, the earliest activation, following stimulus onset, of neurons in successive levels of the cortical hierarchy (Felleman \& Van Essen, 1991). Our finding that STS and hMT $+/ \mathrm{V} 3 \mathrm{~A}$ are activated by coherent motion onset with almost identical latencies suggests that the two areas are processing the information about coherent motion simultaneously and in parallel. STS and hMT+ are known to be involved in extracting structure and form from motion (Todd et al., 2000; Vanduffel et al., 2000; Puce et al., 2003). It may be that the TR-M230 response we measure in these areas reflects aspects of recurrent processing mediated by horizontal and/or feedback connections between and within visual areas - necessary to group the coherently moving dots into a distinct surface (Gilbert, 1993; Payne et al., 1996; Hupé et al., 1998).

In conclusion, our results show that a transition from incoherent to coherent motion elicits a prominent response in $\mathrm{hMT}+/ \mathrm{V} 3 \mathrm{~A}$ and STS approximately $230 \mathrm{~ms}$ after the transition. These responses correlate with the degree of motion coherence.

\section{Acknowledgements}

This study was supported by the Wellcome Trust and by EU's Large-Scale Facility Neuro-BIRCH III at the Brain Research Unit of the Low Temperature Laboratory (HUT). Jane Aspell was supported by a Medical Research Council studentship. We thank all participants in this study. We thank David Bramwell for his many contributions, including writing the stimulus programs. We thank Riitta Hari for her support and collaboration through the EU Neuro-BIRCH III programme, and for helpful comments on earlier versions of the manuscript, Hanna Renvall for comments on the manuscript, and Lauri Parkkonen for expert advice. The MRI scans were obtained at the Department of Radiology, Helsinki University Central Hospital.

\section{Abbreviations}

BOLD, blood oxygenation level dependent; ECD, equivalent current dipole; EEG, electroencephalography; fMRI, functional magnetic resonance imaging; $\mathrm{hMT}+$, human medial temporal area complex; IPS, intraparietal sulcus; MEG, 
magnetoencephalography; MT, medial temporal area; PET, positron emission tomography; RDK, random dot kinematogram; STS, superior temporal sulcus; V3A, V3 accessory area.

\section{References}

Ahlfors, S.P., Simpson, G.V., Dale, A.M., Belliveau, J.W., Liu, A.K., Korvenoja, A., Virtanen, J., Huotilainen, M., Tootell, R.B.H., Aronen, H.J. \& Ilmoniemi, R.J. (1999) Spatiotemporal activity of a cortical network for processing visual motion revealed by MEG and fMRI. J. Neurophysiol., 82, 2545-2555.

Allison, T., Puce, A. \& McCarthy, G. (2000) Social perception from visual cues: role of the STS region. Trends Cogn. Sci., 4, 267-278.

Allman, J.M., Miezin, F.M. \& McGuiness, E. (1985) Direction and velocity specific surrounds in area MT of the owl monkey. Perception, 14, 105-126.

Andersen, R.A. (1997) Neural mechanisms of visual motion perception in primates. Neuron, 18, 865-872.

Anderson, S.J., Holliday, I.E., Singh, K.D. \& Harding, G.F.A. (1996) Localisation and functional analysis of human cortical area V5 using magnetoencephalography. Proc. R. Soc. Lond., B, Biol. Sci., 263, 423431.

Aspell, J.E., Bramwell, D.I., Tanskanen, T. and Hurlbert, A.C. (2000) Neural correlates of coherent visual motion perception measured with MEG. Soc. Neurosci. Abstr., 26, 399.8.

Bach, M. \& Ullrich, D. (1994) Motion adaptation governs the shape of motionevoked cortical potentials. Vis. Res., 34, 1541-1547.

Born, R.T. \& Tootell, R.B.H. (1992) Segregation of global and local motion processing in primate middle temporal visual area. Nature, 357, 497-499.

Braddick, O.J. (1993) Segmentation versus integration in visual-motion processing. TINS, 16, 263-268.

Braddick, O.J., O’Brien, J.M., Wattam-Bell, J., Atkinson, J., Hartley, T. \& Turner, R. (2001) Brain areas sensitive to coherent visual motion. Perception, 30, 61-72.

Braddick, O.J., O’Brien, J.M., Wattam-Bell, J., Atkinson, J. \& Turner, R. (2000) Form and motion coherence activate independent, but not dorsal/ventral segregated networks in the human brain. Curr. Biol., 10, 731-734.

Bradley, D.C. \& Andersen, R.A. (1998) Center-surround antagonism based on disparity in primate area MT. J. Neurosci., 18, 7552-7565.

Britten, K.H., Shadlen, M.N., Newsome, W.T. \& Movshon, J.A. (1992) The analysis of visual motion: a comparison of neuronal and psychophysical performance. J. Neurosci., 12, 4745-4765.

Bruce, C., Desimone, R. \& Gross, C.G. (1981) Visual properties of neurons in a polysensory area in superior temporal sulcus of the macaque. J. Neurophysiol., 46, 369-384.

Dupont, P., Orban, G.A., De Bruyn, B., Verbuggen, A. \& Mortelmans, L. (1994) Many areas in the human brain respond to visual motion. J. Neurophysiol., 72, 1420-1424.

Felleman, D.J. \& Van Essen, D.C. (1991) Distributed hierarchical processing in the primate cerebral cortex. Cereb. Cortex, 1, 1-47.

Gilbert, C.D. (1993) Circuitry, architecture, and functional dynamics of visual cortex. Cereb. Cortex, 3, 373-386.

Grüsser, O.J., Pause, M. \& Schreiter, U. (1990) Vestibular neurones in the parieto-insular cortex of monkeys (Macaca fascicularis): visual and neck receptor responses. J. Physiol. (Lond.), 430, 559-583.

Hämäläinen, M., Hari, R., Ilmoniemi, R.J., Knuutila, J. \& Lounasmaa, O.V. (1993) Magnetoencephalography: Theory, instrumentation, and applications to non-invasive studies of the working human brain. Rev. Mod. Phys., 65, 413-497.

Hari, R. (1990) The neuromagnetic method in the study of the human auditory cortex. Adv. Audiol., 6, 222-282.

Heeger, D.J., Boynton, G.M., Demb, J.B., Seidemann, E. \& Newsome, W.T. (1999) Motion opponency in visual cortex. J. Neurosci., 19, 7162-7174.

Hoffman, M., Dorn, T. \& Bach, M. (1999) Time course of motion adaptation: motion onset visual evoked potentials and subjective estimates. Vis. Res., 39, 437-444.

Hupé, J.M., James, A.C., Payne, B.R., Lomber, S.G., Girard, P. \& Bullier, J. (1998) Cortical feedback improves discrimination between figure and background by V1, V2 and V3 neurons. Nature, 394, 784-787.

Jenkinson, M., Bannister, P., Brady, M. \& Smith, S. (2002) Improved optimisation for the robust and accurate linear registration and motion correction of brain images. Neuroimage, 17, 825-841.

Jenkinson, M. \& Smith, S.M. (2001) A global optimisation method for robust affine registration of brain images. Med. Image Anal., 5, 143-156.
Kuba, M. \& Kubova, Z. (1992) VEPs specific for motion onset. Doc. Opthalmol., 80, 83-89.

Kubova, Z., Kuba, M., Hubacek, J. \& Vit, F. (1990) Properties of visual evoked potentials to onset of movement on a television screen. Doc. Opthalmol., 75, $67-72$.

Lam, K., Kaneoke, Y., Gunji, A., Yamasaki, H., Matsumoto, E., Naito, T. \& Kakigi, R. (2000) Magnetic evoked responses of human extrastriate cortex in the detection of coherent and incoherent motion. Neuroscience, 97, 1-10.

Lamme, V.A.F. \& Roelfsema, P.R. (2000) The distinct modes of vision offered by feedforward and recurrent processing. TINS, 23, 571-579.

Logothetis, N.K., Pauls, J., Augath, M., Trinath, T. \& Oeltermann, A. (2001) Neurophysiological investigation of the basis of the fMRI signal. Nature, 412, 150-157.

McKeefry, D.J., Watson, J.D.G., Frackowiak, R.S.J., Fong, K. \& Zeki, S. (1997) The activity in human areas V1/V2, V3, and V5 during the perception of coherent and incoherent motion. Neuroimage, 5, 1-12.

Merigan, W.H. \& Maunsell, J.H.R. (1993) How parallel are the primate visual pathways? Annu. Rev. Neurosci., 16, 369-402.

Møller, P. \& Hurlbert, A.C. (1997) Motion edges and regions guide image segmentation by colour. Proc. R. Soc. Lond., B, Biol. Sci., 264, 1571-1577.

Movshon, J.A. \& Newsome, W.T. (1996) Visual response properties of striate cortical neurons projecting to area MT in macaque monkeys. J. Neurosci., 16, 7733-7741.

Nakamura, H., Kashii, S., Nagamine, T., Matsui, Y., Hashimoto, T., Honda, Y. \& Shibasaki, H. (2003) Human V5 demonstrated by magnetoencephalography using random dot kinematograms of different coherence levels. Neurosci. Res., 46, 426-433.

Niedeggen, M. \& Wist, E.R. (1998) Motion evoked brain potentials parallel the consistency of coherent motion perception in humans. Neurosci. Lett., 246 , $61-64$.

Niedeggen, M. \& Wist, E.R. (1999) Characteristics of visual evoked potentials generated by motion coherence onset. Brain Res. Cogn. Brain Res., 8, 95-105.

Okada, Y.C., Wu, J. \& Kyuhou, S. (1997) Genesis of MEG signals in a mammalian CNS structure. Electroencephalogr. Clin. Neurophysiol., 103, 474-485.

Oram, M.W. \& Perrett, D.I. (1994) Responses of anterior superior temporal polysensory (STPa) neurons to biological motion stimuli. J. Cogn. Neurosci., 6, 99-116.

Parkkonen, L.T., Simola, J.T., Tuoriniemi, J. \& Ahonen, A.I. (1999) An interference suppression system for multichannel magnetic field detector arrays. In Yoshimoto, T., Kotani, M., Kuriki, S., Karibe, H. \& Nakasato, N., (Eds), Recent Advances in Biomagnetism: Proceedings of the 11th International Conference on Biomagnetism, Tohoku University Press, Sendai, p. 13.

Patzwahl, D.R., Elbert, T., Zanker, J.M. \& Altenmuller, E.O. (1996) The cortical representation of motion in man is inter-individually variable. Neuroreport, 7, 469-472.

Patzwahl, D.R. \& Zanker, J.M. (2000) Mechanisms of human motion perception: combining evidence from evoked potentials, behavioural performance and computational modelling. Eur. J. Neurosci., 12, 273-282.

Payne, B.R., Lomber, S.G., Villa, A.E. \& Bullier, J. (1996) Reversible deactivation of cerebral network components. TINS, 19, 535-542.

Probst, T., Plendl, H., Paulus, W., Wist, E.R. \& Scherg, M. (1993) Identification of the visual motion area (area V5) in the human brain by dipole source analysis. Vis. Res., 93, 345-351.

Puce, A., Allison, T., Bentin, S., Gore, J.C. \& McCarthy, G. (1998) Temporal cortex activation in humans viewing eye and mouth movements. J. Neurosci., 18, 2188-2199.

Puce, A., Syngeniotis, A., Thompson, J.C., Abbott, D.F., Wheaton, K.J. \& Castiello, U. (2003) The human temporal lobe integrates facial form and motion: evidence from fMRI and ERP studies. Neuroimage, 19, 861-869.

Qian, N. \& Andersen, R.A. (1994) Transparent motion perception as detection of unbalanced motion signals. II. Physiology. J. Neurosci., 14, 7367-7380.

Raiguel, S., Van Hulle, M.M., Xiao, D.K., Marcar, V.L. \& Orban, G.A. (1995) Shape and spatial distribution of receptive fields and antagonistic motion surrounds in the middle temporal area (V5) of the macaque. Eur. J. Neurosci., 7, 2064-2082.

Rees, G., Friston, K. \& Koch, C. (2000) A direct quantitative relationship between the functional properties of human and macaque V5. Nature Neurosci., 3, 716-723.

Reppas, J.B., Niyogi, S., Dale, A.M., Sereno, M.I. \& Tootell, R.B.H. (1997) Representation of motion boundaries in retinotopic human visual cortical areas. Nature, 388, 175-179.

Rorden, C. \& Brett, M. (2000) Stereotaxic display of brain lesions. Behav. Neurol., 12, 191-200. 
Scannell, J.W. \& Young, M.P. (1999) Neuronal population activity and functional imaging. Proc. R. Soc. Lond., B, Biol. Sci., 266, 875-881.

Schoenfeld, M.A., Woldorff, M., Duzel, E., Scheich, H., Heinze, H.J. \& Mangun, G.R. (2003) Form-from-motion: MEG evidence for time course and processing sequence. J. Cogn. Neurosci., 15, 157-172.

Sereno, M.I. (1998) Brain mapping in animals and humans. Curr. Opin. Neurobiol., 8, 188-194.

Shulman, G.L., Ollinger, J.M., Akbudak, E., Conturo, T.E., Snyder, A.Z., Petersen, S.E. \& Corbetta, M. (1999) Areas involved in encoding and applying directional expectations to moving objects. J. Neurosci., 19, 9480-9496.

Shulman, G.L., Schwarz, J., Miezin, F.M. \& Petersen, S.E. (1998) Effect of motion contrast on human cortical responses to moving stimuli. J. Neurophysiol., 79, 2794-2803.

Skrandies, W., Jedynak, A. \& Kleiser, R. (1998) Scalp distribution components of brain activity evoked by visual motion stimuli. Exp. Brain Res., 122, 62-70.

Snowden, R.J. \& Braddick, O.J. (1991) The temporal integration and resolution of velocity signals. Vis. Res., 31, 907-914.

Spileers, W., Mangelschots, E., Maes, H. \& Orban, G.A. (1996) Visual evoked potentials elicited by a moving unidimensional noise pattern. Electroencephalogr. Clin. Neurophysiol., 100, 287-298.

Sunaert, S., Fesl, G., Todd, J., Rosier, A., Van Hecke, P. \& Orban, G.A. (2000) Human cortical regions involved in extracting 3D structure from motion depend on stimulus type and transparency. Soc. Neurosci. Abstr., 26, 593.10.

Tadin, D., Lappin, J.S., Gilroy, L.A. \& Blake, R. (2003) Perceptual consequences of centre-surround antagonism in visual motion processing. Nature, 424, 312-315.

Tanaka, K., Kikosaka, K., Saito, H.A., Yukie, M., Fukada, Y. \& Iwai, E. (1986) Analysis of local and wide-field movements in the superior temporal visual areas of the macaque monkey. J. Neurosci., 6, 134-144.

Tootell, R.B.H., Mendola, J.D., Hadjikhani, N.K., Ledden, P.J., Liu, A.K., Reppas, J.B., Sereno, M.I. \& Dale, A.M. (1997) Functional analysis of V3A and related areas in human visual cortex. J. Neurosci., 17, 7060-7078.
Tootell, R.B.H., Reppas, J.B., Kwong, K.K., Malach, R., Born, R.T., Brady, T.J., Rosen, B.R. \& Belliveau, J.W. (1995) Functional analysis of human MT and related visual cortical areas using magnetic resonance imaging. J. Neurosci., 15, 3215-3230.

Uusitalo, M.A. \& Ilmoniemi, R.J. (1997) Signal space projection method for separating EEG or EEG into components. Med. Biol. Eng. Comput., 35, 135-140.

Uusitalo, M.A., Virsu, V., Salenius, S., Nasanen, R. \& Hari, R. (1996) Activation of human V5 complex and rolandic regions in association with moving visual stimuli. Neuroimage, 5, 241-250.

Vaina, L.M. (1994) Functional segregation of color and motion processing in the human visual cortex: clinical evidence. Cereb. Cortex, 4, 555-572.

Vaina, L.M., Solomon, J., Chowdhury, S., Sinha, P. \& Belliveau, J.W. (2001) Functional neuroanatomy of biological motion perception in humans. Proc. Natl Acad. Sci. USA, 98, 11656-11661.

Vanduffel, W.J.M., Beatse, E., Nelissen, K., Tootell, R.B.H., Todd, J.T. \& Orban, G.A. (2000) Areas involved in extracting structure from motion: an fMRI study in the awake fixating monkey. Soc. Neurosci. Abstr., 26, 593.9.

Vanni, S., Uusitalo, M.A., Kiesilä, P. \& Hari, R. (1997) Visual motion activates V5 in dyslexics. Neuroreport, 8, 1939-1942.

Watson, J.D.G., Myers, R., Frackowiak, R.S.J., Hainal, J.V., Woods, R.P., Mazziotta, J.C., Shipp, S. \& Zeki, S. (1993) Area V5 of the human brain: evidence from a combined study using positron emission tomography and magnetic resonance imaging. Cereb. Cortex, 3, 79-94.

Xiao, D.K., Raiguel, S., Marcar, V. \& Orban, G.A. (1997) The spatial distribution of the antagonistic surround of MT/V5 neurons. Cereb. Cortex, 7, 662-677.

Zeki, S.M. (1980) The response properties of cells in the middle temporal area (area MT) of owl monkey visual cortex. Proc. R. Soc. Lond., B, Biol. Sci., 207, 239-248.

Zeki, S.M., Watson, J.D.G., Luseck, C.J., Friston, K.J., Kennard, C. \& Frackowiak, R.S.J. (1991) A direct demonstration of functional properties in human visual cortex. J. Neurosci., 11, 641-649.

Zihl, J., von Cramon, D. \& Mai, N. (1983) Selective disturbance of movement vision after bilateral brain damage. Brain, 106, 313-340. 